\title{
RUNAWAY GROWTH DURING PLANET FORMATION: EXPLAINING THE SIZE DISTRIBUTION OF LARGE KUIPER BELT OBJECTS
}

\author{
Hilke E. Schlichting ${ }^{1,2,5}$ ANd Re'em SARi ${ }^{3,4}$ \\ ${ }^{1}$ Department of Earth and Space Science, UCLA, 595 Charles E. Young Drive East, Los Angeles, CA 90095; hilke@ucla.edu \\ ${ }^{2}$ CITA, University of Toronto, 60 St. George Street, ON, M5S 3H8, Canada \\ ${ }^{3}$ Racah Institute of Physics, Hebrew University, Jerusalem 91904, Israel \\ ${ }^{4}$ California Institute of Technology, MC 130-33, Pasadena, CA 91125 \\ Received 2010 October 12; accepted 2010 December 7; published 2011 January 21
}

\begin{abstract}
Runaway growth is an important stage in planet formation during which large protoplanets form, while most of the initial mass remains in small planetesimals. The amount of mass converted into large protoplanets and their resulting size distribution are not well understood. Here, we use analytic work, that we confirm by coagulation simulations, to describe runaway growth and the corresponding evolution of the velocity dispersion. We find that runaway growth proceeds as follows. Initially, all the mass resides in small planetesimals, with mass surface density $\sigma$, and large protoplanets start to form by accreting small planetesimals. This growth continues until growth by merging large protoplanets becomes comparable to growth by planetesimal accretion. This condition sets in when $\Sigma / \sigma \sim \alpha^{3 / 4} \sim 10^{-3}$, where $\Sigma$ is the mass surface density in protoplanets in a given logarithmic mass interval and $\alpha$ is the ratio of the size of a body to its Hill radius. From then on, protoplanetary growth and the evolution of the velocity dispersion become self-similar and $\Sigma$ remains roughly constant, since an increase in $\Sigma$ by accretion of small planetesimals is balanced by a decrease due to merging with large protoplanets. We show that this growth leads to a protoplanet size distribution given by $N(>R) \propto R^{-3}$, where $N(>R)$ is the number of objects with radii greater than $R$ (i.e., a differential power-law index of 4 ). Since only the largest bodies grow significantly during runaway growth, $\Sigma$ and thereby the size distribution are preserved. We apply our results to the Kuiper Belt, which is a relic of runaway growth where planet formation never proceeded to completion. Our results successfully match the observed Kuiper Belt size distribution, they illuminate the physical processes that shaped it and explain the total mass that is present in large Kuiper Belt objects (KBOs) today. This work suggests that the current mass in large KBOs is primordial and that it has not been significantly depleted. We also predict a maximum mass ratio for Kuiper Belt binaries that formed by dynamical processes of $\alpha^{-1 / 4} \sim 10$, which explains the observed clustering in binary companion sizes that is seen in the cold classical belt. Finally, our results also apply to growth in debris disks, as long as frequent planetesimal-planetesimal collisions are not important during the growth.
\end{abstract}

Key words: celestial mechanics - Kuiper Belt: general - methods: analytical - methods: numerical - minor planets, asteroids: general - planets and satellites: formation

Online-only material: color figures

\section{INTRODUCTION}

Ice giants, the cores of gas giants and protoplanets that later form terrestrial planets, are generally believed to have formed by coagulation from small planetesimals. Understanding the evolution of the size distribution of growing protoplanets, their velocity dispersion, and the interplay between the two is crucial for shedding light onto the planet formation process. Here, we study the runaway growth of protoplanets (e.g., Safronov 1969; Greenberg et al. 1978; Wetherill \& Stewart 1989; Kokubo \& Ida 1996) and their subsequent velocity evolution. Runaway growth can occur when the accretion cross section of protoplanets is enhanced by gravitational focusing. Since, gravitational focusing is strongest for the largest bodies, the radii of larger protoplanets run away from that of smaller. ${ }^{6}$ This leads to a size distribution that develops a tail containing a small number of large protoplanets. It is however not always clear what fraction

\footnotetext{
5 Hubble Fellow.

6 Throughout this paper, we define runaway growth based on the relative growth rates of different sized bodies with respect to each other and emphasize here, given this definition, that the growth of large bodies can still proceed in the runaway regime even while they dominate the viscous stirring (Goldreich et al. 2004b).
}

of the total mass participates in this runaway growth and what protoplanet size distribution such runaway growth gives rise to (Lee 2000; Malyshkin \& Goodman 2001). In this paper we address both of these questions. In the following sections, we discuss runaway growth in the context of the Kuiper Belt, which is an ideal laboratory to test our results, since it is a remnant of the primordial solar system, where planet formation never reached completion. The results, however, also apply to runaway growth during planet formation and growth in the debris disk, as long as gas plays no significant role in the accretion and damping of the velocity dispersion.

The Kuiper Belt consists of a disk of icy bodies located at the outskirts of our planetary system, just beyond the orbit of Neptune and contains some of the least processed bodies in our solar system. Motivated by the discovery of the first Kuiper Belt object (KBO; Jewitt \& Luu 1993) after Pluto and Charon, several groups conducted large-scale surveys to characterize the Kuiper Belt. These efforts led to the discovery of more than 1200 objects in the Kuiper Belt to date. The Kuiper Belt size distribution contains many important clues concerning the formation of KBOs, their effective strength, and their collisional evolution (Dohnanyi 1969; Stern \& Colwell 1997; Davis \& Farinella 1997; Kenyon \& Luu 1999; Pan \& Sari 2005). It also 
provides a snapshot of an earlier stage of planet formation, which was erased elsewhere in the solar system where planet formation proceeded all the way to completion. The cumulative size distribution of KBOs larger than $R \gtrsim 50 \mathrm{~km}$ is described well by a single power law given by

$$
N(>R) \propto R^{1-q},
$$

where $N(>R)$ is the number of objects with radii greater than $R$, and $q$ is the power-law index. Kuiper Belt surveys find that the size distribution for KBOs with radii greater than about $50 \mathrm{~km}$ follows this power law with $q \sim 4$ (e.g., Trujillo et al. 2001; Bernstein et al. 2004; Fuentes \& Holman 2008; Fraser et al. 2008), which implies roughly equal mass per logarithmic mass interval. This size distribution is a relic of the accretion history in the Kuiper Belt and therefore provides valuable insights into the formation of large KBOs $(R \gtrsim 50 \mathrm{~km}$; e.g., Stern 1996; Davis \& Farinella 1997; Kenyon 2002). Observations suggest that there is a break in the power-law size distribution at smaller KBO sizes (e.g., Bernstein et al. 2004; Fuentes \& Holman 2008; Fraser et al. 2008; Schlichting et al. 2009; Fuentes et al. 2010). The break in the size distribution is generally attributed to collisions that break up small KBOs (i.e., $R \lesssim 50 \mathrm{~km}$ ) and modify their size distribution (e.g., Dohnanyi 1969; Kenyon \& Bromley 2004; Pan \& Sari 2005). The KBO size distribution below the break is still poorly constrained, although some encouraging progress has been made recently in probing the abundance of subkilometer-sized KBOs by stellar occultations (e.g., Liu et al. 2008; Schlichting et al. 2009; Bianco et al. 2010).

The work presented in this paper focuses on the size distribution of large $\mathrm{KBOs}(R \gtrsim 50 \mathrm{~km})$, which is well constrained by observations and which sheds light onto the formation of KBOs, protoplanets, and accretion processes that could be ongoing in other debris disks. Numerical coagulation simulations have been successful in reproducing the observed KBO size distribution. Such simulations typically find that the accretion processes of KBOs yield a power-law size distribution with $q \sim 3.8-4.5$ for $10-100 \mathrm{~km}$ and larger objects (Kenyon \& Luu 1999; Kenyon 2002; Kenyon \& Bromley 2004), which is consistent with the observed power-law size distribution. Despite their success, the reason for the actual slope of the distribution has so far not been explained by such simulations. In this paper, we offer an explanation for the slope of the KBO size distribution and for the amount of mass in the large KBOs that are observed in today's Kuiper Belt. Specifically, we find a power-law index of $q \sim 4$ and a total mass in large KBOs of $\sim 10^{-3}$ of the initial planetesimal mass, which is consistent with the current observed mass in the Kuiper Belt. We also make a prediction for the maximum mass ratio of Kuiper Belt binaries that formed by dynamical processes, for example, by three body interactions, and show that our prediction is in good agreement with the observations. Although our work focuses on the Kuiper Belt, the results also apply to early stages of planet formation and protoplanetary growth in debris disks.

Our paper is structured as follows. In Section 2, we analytically describe the growth of large KBOs, including their velocity dispersion and derive the slope of the KBO size distribution. We confirm our analytic results in Section 3 with coagulation simulations. In Section 4, we discuss how semi-collisional accretion, binary mergers, and frequent planetesimal collisions would affect our results. We show that our results on the $\mathrm{KBO}$ growth and velocity dispersion have interesting implications for the formation of Kuiper Belt binaries and predict the maximum mass ratio for binaries that formed by dynamical processes in Section 5. Discussion and conclusions follow in Section 6.

\section{ANALYTICAL TREATMENT}

In order to gain an analytic understanding of the growth processes of large KBOs and the associated velocity evolution we use the "two-groups approximation" (Goldreich et al. 2002, 2004b). The "two-groups approximation" consists of the identification of two groups of objects, small ones, that contain most of the total mass with mass surface density $\sigma$, and large ones, that contain only a small fraction of the total mass with mass surface density $\Sigma \ll \sigma$. We define $\Sigma$ as the mass surface density in a single logarithmic mass interval, that includes the largest bodies formed at a given time. In contrast $\sigma$ is defined as the total mass in small objects.

Within the framework of the "two-groups approximation" we arrive at the following picture for KBO growth. Initially, all the mass is in small bodies. As the small bodies start to accrete each other, large bodies begin to form. To simplify the argument, we only consider the mass surface density of the small and large bodies here, ignoring intermediate size bodies for now. As we show later, the large and small bodies alone determine the velocity dispersion for bodies of all sizes and only the large bodies grow significantly. In the initial stage, $\Sigma$ grows due to the accretion of small bodies. Therefore, the size of the largest bodies and the total mass in large bodies increase with time. During this growth phase, the velocity dispersion of the small bodies increases due to viscous stirring by the large bodies. The velocity dispersion of large bodies is damped by dynamical friction provided by the small bodies. $\Sigma$ continues to grow until the growth of large KBOs by accretion of comparable size objects starts to compete with growth by accretion of small bodies. From then on, $\Sigma$ remains roughly constant in a given logarithmic mass interval, while the size of the large KBOs grows linearly with time. How the KBO growth ended and how exactly the small bodies were lost from the Kuiper Belt are still the subject of ongoing research and are unimportant for the purpose of this paper, and we therefore will not discuss them here further. We confirm the outlined KBO growth analytically and with numerical simulations. We show that the mass ratio, $\Sigma / \sigma$, is not arbitrary but an outcome of $\mathrm{KBO}$ growth.

\subsection{Growth and Velocity Evolution}

Large KBOs viscously stir the small bodies, increasing the small bodies' velocity dispersion $u$. As a result $u$ grows on the same timescale as $R$, as long as the small bodies experience no significant damping by either gas or mutual collisions, which are, most likely, not yet important (see Section 4). We can therefore write the evolution of the small bodies' velocity dispersion as

$$
\frac{1}{u} \frac{d u}{d t} \sim \Omega \frac{\Sigma}{\rho R} \alpha^{-2}\left(\frac{u}{v_{H}}\right)^{-4},
$$

where $\Omega$ is the orbital frequency around the Sun, $R$ is the radius of the large bodies, and $\rho$ is their material density. The Hill velocity of the large bodies is $v_{H}$, which is given by $v_{H}=\Omega R_{H}$, where $R_{H}$ is the Hill radius, $R_{H}=a\left(M / 3 M_{\odot}\right)^{1 / 3}$, where $M_{\odot}$ is the mass of the Sun, $M$ and $a$ are the mass and semi-major axis of the large KBOs. Finally, $\alpha=R / R_{H}$ and is $\sim 10^{-4}$ at the distance of the Kuiper Belt. We assumed in writing the expression for $u$ that $u>v_{H}$, we will verify that this is the 
correct and self-consistent velocity regime for $u$ at the end of this section. Initially, the large bodies grow by accreting the small ones. Their growth rate is given by

$$
\frac{1}{R} \frac{d R}{d t} \sim \Omega \frac{\sigma}{\rho R} \alpha^{-1}\left(\frac{u}{v_{H}}\right)^{-2}
$$

where we assumed that $v<v_{H}$, which we verify later in this section. Equating the rates from Equations (2) and (3) and solving for $u$, we find (Goldreich et al. 2004b)

$$
\frac{u}{v_{H}} \sim\left(\frac{\Sigma}{\sigma \alpha}\right)^{1 / 2}
$$

The velocity $v$ of large KBOs increases due to mutual viscous stirring, but is damped by dynamical friction from the sea of small bodies such that $v<u$. The competition between the stirring and damping can be written as

$$
\frac{1}{v} \frac{d v}{d t} \sim \Omega \frac{\Sigma}{\rho R} \alpha^{-2}\left(\frac{v}{v_{H}}\right)^{-1}-\Omega \frac{\sigma}{\rho R} \alpha^{-2}\left(\frac{u}{v_{H}}\right)^{-4}
$$

Balancing the stirring and damping rates for $v$ and substituting for $u$ from Equation (4) into Equation (5), we find

$$
\frac{v}{v_{H}} \sim \alpha^{-2}\left(\frac{\Sigma}{\sigma}\right)^{3}
$$

Having derived expressions for the large and small bodies' velocity dispersions we now turn to examining the growth in more detail.

Expressions similar to the ones above have been widely used in the literature (e.g., Safronov 1969; Greenberg et al. 1991; Dones \& Tremaine 1993; Rafikov 2003; Goldreich et al. 2004b; Chiang et al. 2007). However, in these works $\Sigma / \sigma$ was treated as a free parameter. Here, we present our method for deriving $\Sigma / \sigma$ and calculate its value during runaway growth.

Within our "two-groups approximation" large bodies have two distinct modes for growth. In the first, they can grow by the accretion of small bodies. In the second, they grow by accreting objects comparable to their own size. The growth rate for large $\mathrm{KBOs}$ is given by

$$
\frac{1}{R} \frac{d R}{d t} \sim \Omega \frac{\sigma}{\rho R} \alpha^{-1}\left(\frac{u}{v_{H}}\right)^{-2}+\Omega \frac{\Sigma}{\rho R} \alpha^{-3 / 2},
$$

where we used the accretion rate corresponding to sub-Hill velocity dispersions for large bodies and the corresponding rate for $u>v_{H}$ for small bodies. The first term in Expression (7) describes the growth of large KBOs by the accretion of small bodies and the second corresponds to the growth of large KBOs by accreting objects of their own size. Substituting the expression for $u$ from Equation (4) we have

$$
\frac{1}{R} \frac{d R}{d t} \sim \Omega \frac{\sigma}{\rho R}\left(\frac{\sigma}{\Sigma}\right)+\Omega \frac{\Sigma}{\rho R} \alpha^{-3 / 2} .
$$

Comparing the two terms in Expression (8) we find that they contribute about equally to the growth of large KBOs, if

$$
\frac{\Sigma}{\sigma} \sim \alpha^{3 / 4} \sim 10^{-3}
$$

Therefore, the growth of large objects will be dominated by the accretion of small bodies, if $\Sigma / \sigma \ll \alpha^{3 / 4}$. If on the other hand
$\Sigma / \sigma \gg \alpha^{3 / 4}$, then accretion of comparable size objects will be the dominant mode of growth provided that $v$ remains less than the Hill velocity. Since initially $\Sigma / \sigma \ll \alpha^{3 / 4}$, it follows that the growth of large KBOs was at the beginning dominated by the accretion of small bodies, as assumed in Equation (3). This mode of growth continues until $\Sigma / \sigma \sim \alpha^{3 / 4}$ at which stage the accretion of comparable size bodies starts to compete with growth by the accretion of small bodies. From then on, large KBOs grow in roughly equal amounts by accreting small bodies and by merging with comparably-sized KBOs. As a result, $\Sigma$ remains constant, since its increase due to the accretion of small bodies is counteracted by a decrease due to the accretion of large KBOs. The radii of large KBOs grow linearly with time (see Equation (8)). We find it very encouraging that the observed mass in large KBOs in each logarithmic size interval estimated from recent Kuiper Belt surveys (e.g., Petit et al. 2008; Trujillo \& Brown 2003; Trujillo et al. 2001) is about $10^{-3}$ of that of the minimum mass solar nebula (MMSN; Hayashi 1981) extrapolated to a heliocentric distance of $40 \mathrm{AU}$. The observed mass in large $\mathrm{KBOs}(R \gtrsim 50 \mathrm{~km})$ is therefore consistent with $\Sigma / \sigma \sim 10^{-3}$ and with the hypothesis that they formed by coagulation in the Kuiper Belt from an MMSN. The growth of KBOs ended in this runaway phase with $\Sigma \sim \alpha^{3 / 4} \sigma \sim 10^{-3} \sigma$. In the context of planet formation, this phase of runaway growth is terminated by the onset of oligarchic growth, when each large body dominates the stirring in its own feeding zone, allowing $\Sigma$ to become comparable to $\sigma$.

Given this understanding of the KBO growth we now show that our choices for the velocity regimes of $u$ and $v$ are selfconsistent with this picture. Substituting $\Sigma / \sigma \sim 10^{-3}$ into Equation (4), we find that $u \sim 3 v_{H}$. This implies that $u$ is about a few times the Hill velocity of the large bodies. We note here that our derivation of the expression for $u$, specifically equating Equations (3) and (2), remains valid even when the large bodies contribute significantly to the growth of large KBOs, because the growth rates due to the accretion of small and large bodies are comparable. Evaluating Equation (6) we find that $v \sim 0.1 v_{H}$. This implies that the velocity dispersion of large bodies was sub-Hill (i.e., $v<v_{H}$ ) during the formation of large 100-kmsized KBOs, while that of the small bodies was super-Hill (i.e., $u>v_{H}$ ). This confirms that we used the correct velocity regime for $u$ and $v$ in the derivation of the KBO accretion rates above, ensuring that our treatment is self-consistent.

Rewriting Equation (9) as

$$
\Sigma \sim \sigma \alpha^{3 / 4} \sim \text { constant }
$$

As we have shown above, $\Sigma$ of the largest bodies is constant with a value of $\sim \alpha^{3 / 4} \sigma$. Once large bodies form, their mass per logarithmic mass interval is, apart from a very brief period (see Section 2.4), preserved. This is because bodies smaller than the largest objects do not grow significantly on the growth timescale of the largest objects, i.e., the growth is in the runaway regime (see Section 2.3). Furthermore, such smaller bodies are also not efficiently consumed by larger objects. Their mass surface density and size distribution are therefore frozen (see Section 2.3). Thus, $\Sigma$ is constant in time, which results in

$$
N(>R) \propto R^{-3}
$$

since $\Sigma \propto N(>R) R^{3}$. This implies a power-law index $q=4$ (see Equation (1)) for large KBOs.

Our work suggests that the growth of large KBOs resulted from the accretion of small and large KBOs in comparable 
amounts and that this mode of growth in the runaway regime gave rise to the observed size distribution of large KBOs. We confirm this result in Section 3 using coagulation simulations. Our findings are consistent with direct observations of the size distribution of large KBOs (Bernstein et al. 2004; Fuentes et al. 2009; Fraser \& Kavelaars 2009) and also agree with results from numerical coagulation simulations that model the growth of KBOs carried out by other groups (e.g., Kenyon \& Luu 1999; Kenyon 2002).

\subsection{Intermediate Sized Bodies: Velocity Dispersion and Growth}

So far, we have only considered two sizes of bodies, small ones and large ones. We now turn our attention to intermediate size bodies with radii $R^{\prime}$, mass surface density $\Sigma^{\prime}$ and velocity dispersion $v^{\prime}$.

In Sections 2.3 and 2.4, we show that $\Sigma^{\prime}$ is of the order $\Sigma$. This is because large bodies form by accreting small bodies until their mass surface density reaches $\Sigma / \sigma \sim \alpha^{3 / 4}$. From then on, the mass surface density remains roughly constant in a given logarithmic mass interval. $\Sigma \sim \Sigma^{\prime}$ because once larger bodies form, the velocity dispersion of the now intermediate sized bodies quickly grows to super-Hill velocities (i.e., $v^{\prime}>v_{H}$ ), which implies that such intermediate sized bodies do not grow significantly on the growth timescale of the largest KBOs and that they are not consumed efficiently by the larger objects. The size distribution of such intermediate sized bodies and $\Sigma^{\prime}$ therefore remain constant. During the brief period over which $v^{\prime}<v_{H}$, intermediate sized bodies are efficiently accreted by the largest objects, but we show in Section 2.4 that this phase is very short and that it extends over less than a factor of two in radius. As a result, $\Sigma^{\prime}$ is of the order of, but slightly smaller than, $\Sigma$.

\subsection{Intermediate-sized Bodies with $v^{\prime}>v_{H}$}

There are three different velocity regimes for intermediatesized bodies that we have to consider separately. In the first regime, the intermediate-sized bodies' velocity dispersion exceeds the Hill velocity of the largest bodies, i.e., $v^{\prime}>v_{H}$, and the bodies themselves are sufficiently small such that their velocity dispersion is not efficiently damped by dynamical friction. In other words, the dynamical friction timescale for these intermediate-sized bodies exceeds the growth timescale of the large bodies. The velocity dispersion of these bodies is therefore dominated by gravitational stirring from the large bodies and it grows on the same timescales as the size of the large bodies. This implies that $v^{\prime} \sim u$ :

$$
\frac{v^{\prime}}{v_{H}} \sim \frac{u}{v_{H}} \sim\left(\frac{\Sigma}{\sigma \alpha}\right)^{1 / 2} \sim \alpha^{-1 / 8}
$$

where we substituted $\Sigma / \sigma \sim \alpha^{3 / 4}$ from Equation (9) in the last step. Equating the dynamical friction timescale for bodies of size $R^{\prime}$ to the growth timescale of the large bodies and solving for $R^{\prime}$, we have

$$
\frac{R^{\prime}}{R} \sim \frac{\Sigma}{\sigma} \alpha^{-1 / 2} \sim \alpha^{1 / 4}
$$

This implies that $v^{\prime} \sim u$ for intermediate-sized bodies with $R^{\prime} \sim \alpha^{1 / 4} R \sim 0.1 R$ and smaller. For bodies above this size, damping by dynamical friction is important.
In the second velocity regime, which applies for bodies larger than $\alpha^{1 / 4} R \sim 0.1 R$ and that have $v^{\prime}>v_{H}$, the evolution of the velocity dispersion is dominated by gravitational stirring from the large bodies and damping by dynamical friction generated by the small bodies. This yields a velocity dispersion that is governed by

$$
\frac{1}{v^{\prime}} \frac{d v^{\prime}}{d t} \sim \Omega \frac{\Sigma}{\rho R} \alpha^{-2}\left(\frac{v^{\prime}}{v_{H}}\right)^{-4}-\Omega \frac{\sigma}{\rho R^{\prime}} \alpha^{-2}\left(\frac{u}{v_{H}^{\prime}}\right)^{-4} .
$$

Balancing the stirring and damping rates and substituting $v_{H}^{\prime}=v_{H}\left(R^{\prime} / R\right)$, we find that $v^{\prime}$ is given by

$$
\frac{v^{\prime}}{v_{H}} \sim \alpha^{-1 / 2}\left(\frac{\Sigma}{\sigma}\right)^{3 / 4}\left(\frac{R^{\prime}}{R}\right)^{-3 / 4} \sim \alpha^{1 / 16}\left(\frac{R^{\prime}}{R}\right)^{-3 / 4},
$$

where we substituted for $u$ from Equation (4) and used $\Sigma / \sigma \sim$ $\alpha^{3 / 4}$ in the last step. Equation (15) yields that $v^{\prime} \sim v_{H}$ for KBOs with $R^{\prime} \sim \alpha^{1 / 12} R \sim 0.5 R$. Therefore, $v^{\prime}>v_{H}$ for bodies with $R^{\prime} \lesssim 0.5 R$. This implies that KBOs with radii smaller than $\sim 0.5 R$ have super-Hill velocities (i.e., $v^{\prime}>v_{H}$ ) and those with radii larger than $\sim 0.5 R$ will have sub-Hill velocities (i.e., $\left.v^{\prime}<v_{H}\right)$.

The growth of intermediate-sized bodies that have $v^{\prime}>v_{H}$ and $R^{\prime}>\alpha^{1 / 4} R \sim 0.1 R$ is given by

$$
\begin{aligned}
\frac{1}{R^{\prime}} \frac{d R^{\prime}}{d t} \sim & \Omega \frac{\sigma}{\rho R}\left(\frac{R^{\prime}}{R}\right)\left(\frac{u}{v_{H}}\right)^{-2} \alpha^{-1} \\
& +\Omega \frac{\Sigma^{\prime}}{\rho R}\left(\frac{R^{\prime}}{R}\right)\left(\frac{v^{\prime}}{v_{H}}\right)^{-2} \alpha^{-1} .
\end{aligned}
$$

Substituting for $u$ from Equation (4) and using again the result that $\Sigma / \sigma \sim \alpha^{3 / 4}$, we can write the above expression as

$$
\frac{1}{R^{\prime}} \frac{d R^{\prime}}{d t} \sim \Omega \frac{\Sigma}{\rho R}\left(\frac{R^{\prime}}{R}\right) \alpha^{-3 / 2}+\Omega \frac{\Sigma^{\prime}}{\rho R}\left(\frac{R^{\prime}}{R}\right)\left(\frac{v^{\prime}}{v_{H}}\right)^{-2} \alpha^{-1},
$$

where the first term corresponds to growth by the accretion of small bodies and the second term to growth by mergers of comparable sized bodies. Since $v^{\prime}>v_{H}$, we find when comparing the magnitude of the two growth terms in Equation (17) that the growth is dominated by the accretion of small bodies. We assumed that $\Sigma^{\prime}$ is of the order of $\Sigma$, which we confirm in Section 2.4. Intermediate-sized bodies with $R^{\prime} \lesssim 0.5 R$ therefore grow predominantly by accreting small bodies and their growth rate is reduced by a factor of $\left(R^{\prime} / R\right)$ compared to bodies of size $R$. This implies that bodies of size $R^{\prime}$ do not get the chance to grow, compared to the growth time scale of bodies of size $R$.

These intermediate-sized bodies do not contribute significantly to the growth of the large bodies. When examining the contributions from bodies with $v^{\prime}>v_{H}$ to the growth of bodies of size $R$, we have

$$
\frac{1}{R} \frac{d R}{d t} \sim \Omega \frac{\Sigma}{\rho R} \alpha^{-3 / 2}+\Omega \frac{\Sigma^{\prime}}{\rho R}\left(\frac{v^{\prime}}{v_{H}}\right)^{-2} \alpha^{-1},
$$

where the first and second terms correspond to growth by merging with bodies of sizes $R$ and $R^{\prime}$, respectively. Since the first term in Equation (18) exceeds the second, large bodies grow predominantly by accreting small bodies and large bodies. Intermediate-sized bodies with $v^{\prime}>v_{H}$ are not important for 
this growth. Furthermore, such intermediate-sized bodies are only inefficiently accreted by bodies of size $R$. This is apparent when examining the rate of change of their surface density due to accretion onto bodies of size $R$ :

$$
\frac{1}{\Sigma^{\prime}} \frac{d \Sigma^{\prime}}{d t} \sim \Omega \frac{\Sigma}{\rho R}\left(\frac{v^{\prime}}{v_{H}}\right)^{-2} \alpha^{-1} .
$$

Comparing Equation (19) with Equation (7), we find that $\Sigma^{\prime}$ does not change significantly on the growth timescale of the large bodies, which implies that their surface density is not altered due to accretion into large bodies. As a result, the mass surface density per logarithmic mass interval of intermediatesized bodies with $v^{\prime}>v_{H}$ remains constant in time.

\subsection{Intermediate-sized Bodies with $v^{\prime}<v_{H}$}

Intermediate-sized bodies that are about half the size of the large bodies and larger will have a velocity dispersion that is smaller than the large bodies' Hill velocity. The evolution of the velocity dispersion of such intermediate-sized bodies is determined by gravitational stirring from large bodies and damping by dynamical friction generated by the small bodies. The expression for the evolution of $v^{\prime}$ can be written as

$$
\frac{1}{v^{\prime}} \frac{d v^{\prime}}{d t} \sim \Omega \frac{\Sigma}{\rho R} \alpha^{-2}\left(\frac{v^{\prime}}{v_{H}}\right)^{-1}-\Omega \frac{\sigma}{\rho R^{\prime}} \alpha^{-2}\left(\frac{u}{v_{H}^{\prime}}\right)^{-4} .
$$

Balancing the stirring and damping rates and substituting $v_{H}^{\prime}=v_{H}\left(R^{\prime} / R\right)$, we can write $v^{\prime}$ as

$$
\frac{v^{\prime}}{v_{H}} \sim \alpha^{-2}\left(\frac{\Sigma}{\sigma}\right)^{3}\left(\frac{R^{\prime}}{R}\right)^{-3} \sim \alpha^{1 / 4}\left(\frac{R^{\prime}}{R}\right)^{-3},
$$

where we substituted for $u$ from Equation (4) and used $\Sigma / \sigma \sim$ $\alpha^{3 / 4}$ in the last step. Strictly speaking, we should multiply the right-hand side of Equation (21) by a logarithmic factor, which is given by $3 \times \log \left(R / R_{*}\right)$, where $R_{*}$ is equal to $R^{\prime}$, if $v^{\prime}<v_{H}^{\prime}$. If $v^{\prime}>v_{H}^{\prime}$ then $R_{*}$ is the radius that corresponds to bodies with a velocity dispersion that is less than or equal to $v_{H}^{\prime}$. The base of the logarithm is the same as the base of the logarithmic mass interval over which $\Sigma$ is defined. This logarithmic factor arises because all bodies larger than or equal to $R^{\prime}$ that have a velocity dispersion less than $v_{H}^{\prime}$ contribute to the gravitational stirring for $q=4$. This is a key difference compared to the super-Hill velocity regime where the stirring is dominated by the large bodies alone.

The growth of intermediate-sized bodies with $R^{\prime}>\alpha^{1 / 12} R \sim$ $0.5 R$ is given by

$$
\frac{1}{R^{\prime}} \frac{d R^{\prime}}{d t} \sim \Omega \frac{\sigma}{\rho R}\left(\frac{R^{\prime}}{R}\right)\left(\frac{u}{v_{H}}\right)^{-2} \alpha^{-1}+\Omega \frac{\Sigma^{\prime}}{\rho R^{\prime}} F_{\mathrm{acc}}
$$

where

$$
F_{\mathrm{acc}}= \begin{cases}\alpha^{-1}\left(\frac{v^{\prime}}{v_{H}^{\prime}}\right)^{-2} & \text { if } v^{\prime}>v_{H}^{\prime} \\ \alpha^{-3 / 2} & \text { if } v^{\prime}<v_{H}^{\prime}\end{cases}
$$

The radius, $R_{v_{H}^{\prime}}$, for which $v^{\prime} \sim v_{H}^{\prime}$ can be found from Equation (21) when including the logarithmic factor and it is given by $R_{v_{H}^{\prime}} / R \sim \alpha^{1 / 16}\left(3 \times \log \left[R / R_{v_{H}^{\prime}}\right]\right)^{1 / 4}$. The $v^{\prime}>v_{H}^{\prime}$ regime given in Equation (22) yields the same expression for the accretion rate as given by Equation (17). The growth in this case, as shown above, is dominated by the accretion of small bodies. In the second case, with $v^{\prime}<v_{H}^{\prime}$, the rate of growth of bodies with radii $R^{\prime}$ is

$$
\frac{1}{R^{\prime}} \frac{d R^{\prime}}{d t} \sim \Omega \frac{\Sigma}{\rho R}\left(\frac{R^{\prime}}{R}\right) \alpha^{-3 / 2}+\Omega \frac{\Sigma^{\prime}}{\rho R^{\prime}} \alpha^{-3 / 2},
$$

where we substituted again for $u$ from Equation (4) and used $\Sigma / \sigma \sim \alpha^{3 / 4}$. The first term corresponds to growth by the accretion of small bodies and the second to growth by merger of comparable sized bodies. Comparing the magnitude of the two growth terms in Equation (24) we find that, unlike in the regime discussed above, the growth of bodies with radius $R^{\prime}$ is dominated by the accretion of similar sized bodies rather than by the accretion of small bodies, again assuming that $\Sigma^{\prime}$ is of the order of $\Sigma$, which we show below. We note here that similar to the sub-Hill velocity excitation rate, the mass accretion rate that corresponds to the accretion of comparable sized bodies should have been multiplied by a logarithmic factor, which is given by $3 \times \log \left[R^{\prime} / R_{v_{H}^{\prime}}\right]$. The maximum value for $R^{\prime} / R_{v_{H}^{\prime}}$ is given by $\alpha^{-1 / 12} \sim 2$. Strictly speaking, we should also have included this logarithmic factor in the derivation of Equation (9). However, since this logarithmic factor only provides a small correction it does not warrant to be included here, since we have been neglecting factors of order unity throughout. Although the overall growth proceeds in the runaway regime (i.e., $\left.(d \log [R] / d t) /\left(d \log \left[R^{\prime}\right] / d t\right)=R / R^{\prime}\right)$, large bodies with $v^{\prime}<v_{H}^{\prime}$ grow in an orderly fashion with respect to each other if $q=4$, which implies that their radii converge. This leads to a steepening in the size distribution at very large radii, but only for bodies with radii that have a corresponding velocity dispersion such that $v^{\prime}<v_{H}^{\prime}$, which corresponds to less than a factor of two in radius. The largest effect this can have is to reduce $\Sigma$ by a factor of $\sim 2$, such that $\Sigma^{\prime} \sim 0.5 \Sigma$, since orderly growth will cease when $\Sigma / R \sim \Sigma^{\prime} / R^{\prime}$.

Intermediate-sized bodies with $v^{\prime}<v_{H}$ significantly contribute to the growth of large bodies, since

$$
\frac{1}{R} \frac{d R}{d t} \sim \Omega \frac{\Sigma}{\rho R} \alpha^{-3 / 2}+\Omega \frac{\Sigma^{\prime}}{\rho R} \alpha^{-3 / 2},
$$

where the first and second terms correspond to growth by merging with bodies of sizes $R$ and $R^{\prime}$, respectively. This implies that for $q=4$, i.e., $\Sigma \sim \Sigma^{\prime}$, large bodies of size $R$ grow at comparable rates by accreting bodies of their own size and bodies of size $R^{\prime} \gtrsim 0.5 R$. Because these intermediate-sized bodies are efficiently accreted by large bodies, their surface density is altered at a rate

$$
\frac{1}{\Sigma^{\prime}} \frac{d \Sigma^{\prime}}{d t} \sim \Omega \frac{\Sigma}{\rho R} \alpha^{-3 / 2}
$$

Comparing Equation (26) with Equation (7), we find that $\Sigma^{\prime}$ changes on the order of the growth timescale of the large bodies. As a result, the mass surface density per logarithmic mass interval of intermediate-sized bodies with $v^{\prime}<v_{H}$ gets depleted, because such bodies are efficiently consumed by large bodies. However, since the size range is very small, such bodies are only depleted by factor of a few before their velocity dispersions become super-Hill at which point they are no longer efficiently accreted. The orderly growth of bodies with $v^{\prime}<v_{H}^{\prime}$ relative to each other and the efficient consumption of bodies with $v^{\prime}<v_{H}$ are responsible for the steepening of the KBO size distribution seen at $R^{\prime}>R_{v_{H}^{\prime}}$ in Figure 2 . 


\section{COAGULATION SIMULATIONS}

\subsection{Model}

The aim of our coagulation simulation is to test our analytic results outlined above. Its purpose is to capture the dominating physical processes that give rise to the $\mathrm{KBO}$ size distribution. We attempt by no means to present the most detailed or precise KBO formation simulation, since several such works already exist in the current literature (e.g., Kenyon \& Luu 1999; Kenyon 2002; Kenyon \& Bromley 2004). We therefore neglect factors of order unity in the accretion, stirring, and damping rates. In addition, we neglect effects of gas damping and possible dynamical stirring from Neptune. We investigate the $\mathrm{KBO}$ growth in a single annulus located at roughly $40 \mathrm{AU}$ from the Sun with a width of about $10 \mathrm{AU}$. Most of our simulations start with a total mass of about 40 Earth masses in small planetesimals, which corresponds to $\sigma \sim 0.65 \mathrm{~g} \mathrm{~cm}^{-2}$. This mass surface density is consistent with extrapolations of the MMSN (Hayashi 1981) to $40 \mathrm{AU}$, after it has been enhanced about sixfold as required for the formation of Uranus and Neptune (e.g., Goldreich et al. 2004a; Dodson-Robinson \& Bodenheimer 2010). We follow the mass growth and the evolution of the velocity dispersion of the KBOs using Safronov's statistical approach (Safronov 1969). Since we are primarily concerned with the initial growth phase, we assume here that all collisions lead to accretion. This assumption is justified during the initial KBO growth and remains justified even once the planetesimal's velocity dispersion has been excited above its own escape velocity as long as the growth timescale of the large KBOs is short compared to the planetesimal-planetesimal collision time. This condition is generally fulfilled, if the initial planetesimals are of the order of a kilometer in size or larger, since, for 1-km-sized bodies, the planetesimal-planetesimal collision time is comparable to the formation time of Pluto. We therefore neglect the effect of destructive planetesimal collisions here, but we discuss how they would effect our results, had they been important, in Section 4.

Below we give the relevant stirring, damping, and accretion rates that determine the growth and velocity evolution in the Kuiper Belt. A detailed derivation of the these rates can, for example, be found in Goldreich et al. (2004b). The accretion rate between bodies of two different mass bins is given by

$$
\begin{aligned}
\mathcal{R}_{\text {coll }} \sim & \Omega \frac{N_{B} \Sigma_{s}}{\rho R_{B}}\left(\frac{M_{B}}{M_{S}}\right) \\
& \times \begin{cases}1 & v_{\mathrm{esc}_{\mathrm{B}}}<v_{\text {rel }} \\
\alpha^{-1}\left(\frac{v_{\text {rel }}}{v_{H_{B}}}\right)^{-2} & v_{H_{B}}<v_{\text {rel }}<v_{\mathrm{esc}_{B}} \\
\alpha^{-3 / 2} & v_{\text {rel }}<v_{H_{B}},\end{cases}
\end{aligned}
$$

where the subscripts " $s$ " and " $B$ " correspond to the mass bin with the smaller and larger bodies, respectively. The number of bodies in a given mass bin is given by $N$ and $v_{\text {rel }}$ is the relative velocity, which was approximated by $v_{\text {rel }}=\operatorname{Max}\left[\mathrm{v}_{\mathrm{s}}, \mathrm{v}_{\mathrm{B}}\right]$. Note, we quote here the sub-Hill accretion rate applicable to large objects, since the velocity dispersion of the small bodies is super-Hill throughout the growth.

The corresponding velocity evolution for each mass bin is dominated by the following processes. The velocity dispersion of the mass bin that corresponds to the smaller objects is viscously stirred by the object in the larger mass bin at a rate given by

$$
\frac{1}{v_{s}} \frac{d v_{s}}{d t} \sim \Omega \frac{\Sigma_{B}}{\rho R_{B}} \times \begin{cases}1 & v_{\mathrm{esc}_{\mathrm{B}}}<v_{\text {rel }} \\ \alpha^{-2}\left(\frac{v_{\mathrm{rel}}}{v_{H_{B}}}\right)^{-4} & v_{H_{B}}<v_{\text {rel }}<v_{\mathrm{esc}_{\mathrm{B}}} \\ \alpha^{-2}\left(\frac{v_{\text {rel }}}{v_{H_{B}}}\right)^{-1} & v_{\text {rel }}<v_{H_{B}} .\end{cases}
$$

The velocity dispersion of the bodies in the larger of the two mass bin is in turn damped by the smaller bodies. The damping rate of the large bodies velocity dispersion is given by

$$
\frac{1}{v_{B}} \frac{d v_{B}}{d t} \sim-\Omega \frac{\Sigma_{s}}{\rho R_{B}} \times \begin{cases}1 & v_{\mathrm{esc}_{\mathrm{B}}}<v_{\text {rel }} \\ \alpha^{-2}\left(\frac{v_{\text {rel }}}{v_{H_{B}}}\right)^{-4} & v_{H_{B}}<v_{\text {rel }}<v_{\mathrm{esc}_{\mathrm{B}}} \\ \alpha^{-2} & v_{\text {rel }}<v_{H_{B}} .\end{cases}
$$

We implemented the above equations in our coagulation code and followed the growth and velocity evolution of the various mass bins.

\subsection{Results}

\subsubsection{KBO Growth and Velocity Evolution}

As initial conditions we start with an equivalent of about 40 Earth masses, in 1-km-sized planetesimals with an initial velocity dispersion of three times their Hill velocity. We follow the KBO growth and velocity evolution of the different mass bins for 70 Myr. The results of our coagulation simulation are shown in Figures 1-3.

Figure 1 shows the evolution of the cumulative mass distribution, the cumulative number distribution, and the velocity dispersion during $\mathrm{KBO}$ growth as a function of time. It is apparent from Figure 1 that the growth and velocity evolution become self-similar once $\Sigma \sim \alpha^{3 / 4} \sigma \sim 10^{-3} \sigma$, i.e., the shapes of the size distribution and velocity distribution remain unchanged, while the maximum KBO size continues to grow.

The two upper panels of Figures 1 and 2 show that the size distribution of large $\mathrm{KBOs}(R \gtrsim 50 \mathrm{~km})$ indeed follows a power law with $q=4$ as predicted by our analytic treatment in Section 2. This implies a roughly equal amount of mass per logarithmic mass bin for large KBOs. Moreover, the middle panels of Figures 1 and 2 show that the mass ratio of large-tosmall KBOs, i.e., $\Sigma / \sigma$, found in our coagulation simulation agrees very well with our analytic prediction that $\Sigma / \sigma \sim$ $\alpha^{-3 / 4} \sim 10^{-3}$. The simulations confirm our analytic results and suggest that the total mass in large objects that we see in the Kuiper Belt is not arbitrary but an outcome of the KBO growth and that it is roughly $10^{-3}$ of the initial planetesimals mass. This result is in excellent agreement with the actual observed mass in large KBOs and formation from an MMSN-type disk. Our work therefore suggests that the Kuiper Belt did not contain two-tothree orders of magnitude more mass in large KBOs as has been proposed by some models (Weidenschilling 2002; Tsiganis et al. 2005).

Figure 3 shows a comparison of the velocity dispersion from our coagulation simulation and our analytic results derived in Section 2 . It displays very good agreement between the velocity dispersion that we derived analytically for the various size regimes, and the results from our coagulation simulation.

Figures 1-3 show that our analytic work captures the essential features of KBO growth and that analytic theory and the 

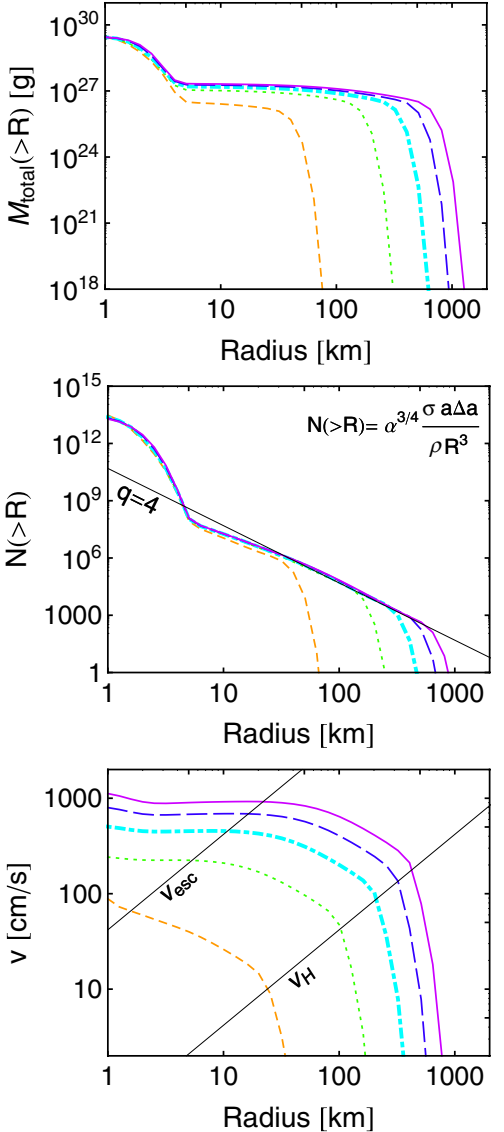

Figure 1. Evolution of the cumulative mass distribution, the cumulative number distribution, and the velocity dispersion during $\mathrm{KBO}$ growth as a function of time at $3 \times 10^{7} \mathrm{yr}$ (dashed orange line), $4 \times 10^{7} \mathrm{yr}$ (dotted green line), $5 \times 10^{7} \mathrm{yr}$ (dot-dashed turquoise line), $6 \times 10^{7} \mathrm{yr}$ (long-dashed blue line), and $7 \times 10^{7} \mathrm{yr}$ (solid purple line). The growth of the large $\mathrm{KBOs}(R \geq 50 \mathrm{~km})$ becomes selfsimilar from $4 \times 10^{7} \mathrm{yr}$ onward, i.e., the shapes of the size distribution and of the velocity distribution remain unchanged, but the $\mathrm{KBO}$ size and velocity continue to grow with time. The slope of the large $\mathrm{KBO}$ size distribution is $q \sim 4$. A power-law index of $q=4$ corresponds to a horizontal line in the top panel of this figure. The thin black line in the middle panel of this figure represents the KBO size distribution with $q=4$, as predicted by our analytic theory and its expression is given in the top right-hand corner. The bottom panel shows the evolution of velocity dispersion during $\mathrm{KBO}$ growth. The escape velocity and Hill velocity (assuming a KBO material density of $1 \mathrm{~g} \mathrm{~cm}^{-3}$ ) are given as a function of size by the upper and lower thin black lines, respectively. We refer the reader to Figure 3 for a detailed comparison between our analytic theory and the velocity evolution found from our simulations. Our analytic theory and the numerical coagulation results are in excellent agreement.

(A color version of this figure is available in the online journal.)

numerical coagulation results are in excellent agreement. We are able to successfully explain the slope $(q=4)$ and amplitude $\left(\Sigma \sim \alpha^{-3 / 4} \sigma\right)$ of the large KBO size distribution and the evolution of the velocity dispersion in the various velocity and size regimes.

\subsubsection{Initial Planetesimal Size Distribution and Sizes}

We performed an additional set of coagulation simulations with different initial conditions. In the first set, we started with most of the mass in small 1-km-sized objects, just as before, but added a second population of larger 10-km-sized KBOs that contained $10^{-3}$ of the total mass. All bodies were started with a velocity dispersion equal to three times their Hill velocity, and we followed their growth and velocity evolution. Figure 4 shows the result of this growth (points) and a comparison with

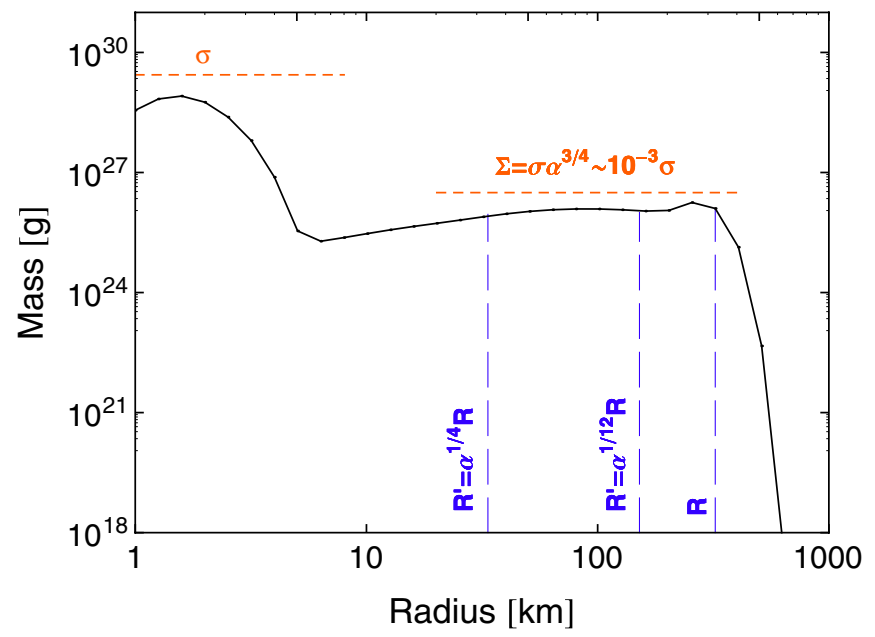

Figure 2. KBO size distribution as a function of radius at $5 \times 10^{7} \mathrm{yr}$ from the same coagulation simulations as shown in Figure 1. The $y$-axis corresponds to the mass in a given $\log _{2}$ mass interval. The total mass in small planetesimals is given by $\sigma$, and its values is given by the orange dashed line drawn below its symbol. Similarly, the mass in large KBOs in a given $\log _{2}$ mass interval is denoted by $\Sigma$ and its predicted value from Section 2 is given by orange dashed lines drawn below it. The value for $\Sigma$ from our analytic work and numerical coagulation simulation agrees within a factor of two. This agreement can even be improved, if we account for the fact that all logarithmic mass bins with $v^{\prime}<v_{H}$ contribute to the growth of the largest bodies about equally (see discussion following Equation (24) in Section 2), which would reduce the predicted value of $\Sigma$ somewhat. The dashed vertical lines mark the radii separating the different velocity dispersion regimes, as discussed in Section 2.

(A color version of this figure is available in the online journal.)

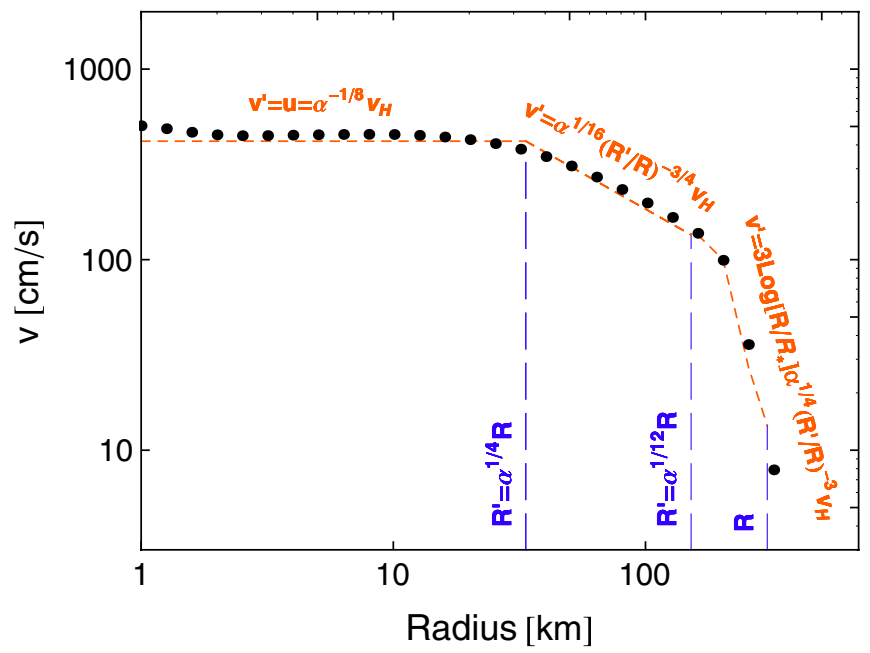

Figure 3. Velocity dispersion as a function of radius at $5 \times 10^{7} \mathrm{yr}$. The results from our coagulation simulation are given by the black points, the dashed orange lines are our analytic predictions for the velocity dispersion with their equations given above each segment. The dashed vertical lines mark the radii separating the different velocity dispersion regimes, as discussed in Section 2.

(A color version of this figure is available in the online journal.)

the cumulative mass distribution at various times for KBOs that grew from a single population of 1-km-sized planetesimals (lines). The similarity between the two distributions is striking. The same power law for the large KBO size distribution emerges and the mass ratio in large and small KBOs becomes also the same in both simulations. These results highlight the power of the "two groups approximation" that we used to derive the analytic results in Section 2 and validate our assertion that the growth of large KBOs develops toward a state where small 


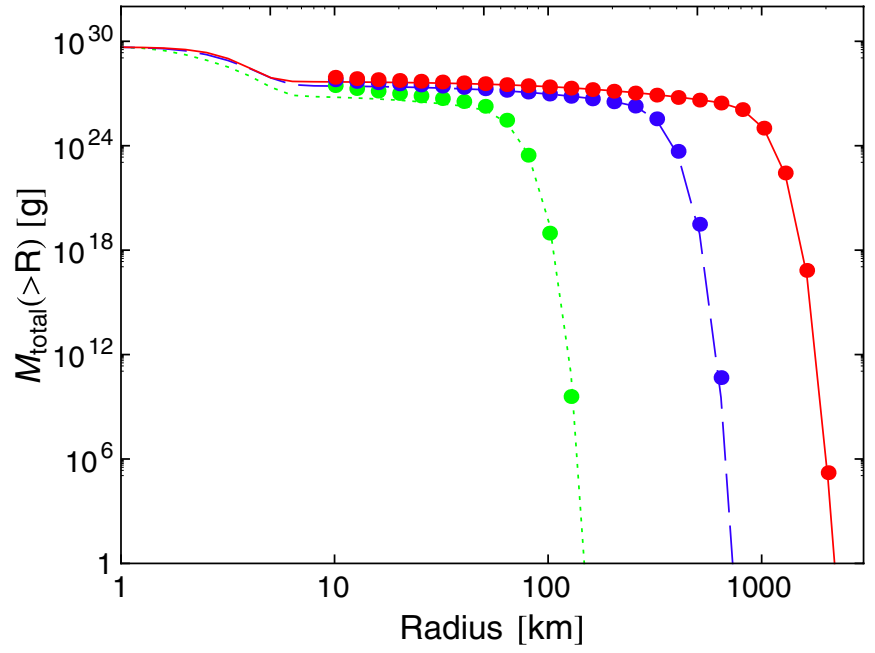

Figure 4. Direct comparison of KBO growth from two different initial conditions. The lines correspond to starting with only $1-\mathrm{km}$-sized bodies. The points represent the KBO growth that resulted when starting with the same total mass as before, but in addition to having most of the mass in 1-km-sized objects we added a second population of $10-\mathrm{km}$-sized KBOs that contain $10^{-3}$ of the total mass. The cumulative mass distribution of the small objects is omitted here, since it is the size distribution of large KBOs that we want to compare here. The agreement between the two simulations is striking. The shape, amplitude (i.e., the ratio of $\Sigma / \sigma$ ), and power-law slope is therefore not a result of the initial conditions but represents an "equilibrium state" that the system evolves to. The dotted green line corresponds to a time of about $3 \times 10^{7} \mathrm{yr}$ since the start of the simulation, whereas the green points correspond to only about $10^{6} \mathrm{yr}$ since the start of the simulation. Due to the different initial conditions it took a different amount of time to grow to 50-km-sized KBOs, but the growth timescale once these 50-km-sized KBOs have formed (green dashed line and points) becomes the same. In other words, it takes the same amount of time to grow from 50-km-sized KBOs to Pluto-sized objects irrespective of the initial conditions, once 50-km-sized KBOs have formed. The blue dashed line and points and the red solid line and points correspond to $1.5 \times 10^{7}$ and to $5 \times 10^{7}$ $\mathrm{yr}$ since the formation of objects that are represented by the green dashed lines and points, respectively.

(A color version of this figure is available in the online journal.)

and large bodies contribute about equally to the growth. The overall growth timescale to reach Pluto-mass objects differs in these two simulations. Starting from 40 Earth masses in small planetesimals, it takes in our simulations about 70-80 Myr to form Pluto-sized objects if initially all KBOs are $1 \mathrm{~km}$ in radius but only $40-50 \mathrm{Myr}$ if, in addition to the $1-\mathrm{km}$-sized objects, there also existed a small population of $10-\mathrm{km}$-sized KBOs. The difference in the growth time results from the timescale it takes 1-km-sized objects to grow into $10-\mathrm{km}$-sized bodies, if none are already present. When only comparing the growth timescale from 50-km-sized objects to $1000-\mathrm{km}$-sized objects, we find that they are the same in the two scenarios (see Figure 4), confirming that it is the initial growth of objects to tens of kilometers in size that give rise to the overall difference in Pluto formation time between the two simulations. The final shape of the KBO size distribution and the total mass in large KBOs (as a fraction of the initial mass) is therefore independent of the initial size distribution of the planetesimals. We also find the same KBO size distribution and overall mass in large KBOs, if the initial planetesimals have a power-law size distribution with various power-law indexes and radii ranging from 1 to $10 \mathrm{~km}$. In addition, we confirm that our results are independent of the details of the initial planetesimal velocity dispersion, as long as it is below their escape velocity.

We also performed a second set of coagulation simulations, which we started with the same total planetesimal mass but with

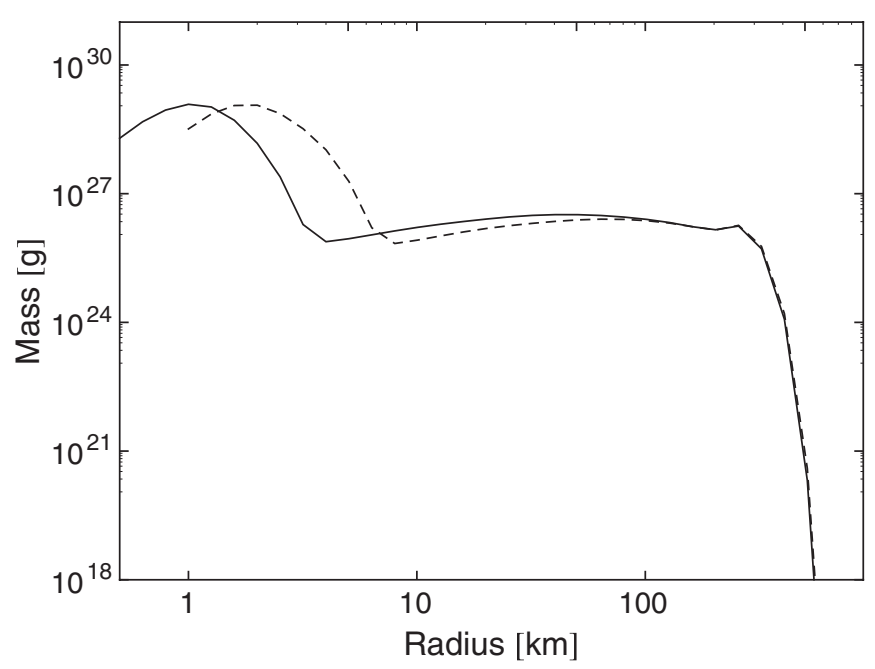

Figure 5. Comparison of KBO growth from two different initial planetesimal sizes. In each simulation, all planetesimals started out with one size. The solid and dashed lines correspond to an initial planetesimal size of $500 \mathrm{~m}$ and $1 \mathrm{~km}$, respectively. The mass distribution is shown after a growth time of $\sim 5 \times 10^{7} \mathrm{yr}$. The $y$-axis corresponds to the mass in a given $\log _{2}$ mass interval. The shape of the size distribution does not depend on the initial planetesimal size, as long as the planetesimal-planetesimal collision timescale exceeds the KBO growth timescale, such that dynamical cooling and possible mass loss due to frequent planetesimal collisions can be neglected.

an initial planetesimal size of $500 \mathrm{~m}$ in radius rather than $1 \mathrm{~km}$. Figure 5 shows that the shape of the size distribution does not depend on the initial planetesimal size. This result remains valid as long as the initial planetesimal size is large enough such that the planetesimal collision timescale exceeds the KBO growth timescale.

These results confirm earlier findings by Kenyon \& Luu (1999), who note that the power-law slope for large objects is remarkably independent of the input parameters and initial conditions. Our work offers an explanation of why this is so. Since the size distribution of large KBOs reaches an equilibrium state that evolves self-similarly, the signature of the initial planetesimal size distribution and their initial velocity dispersion are erased. The slope of the KBO size distribution and the formation timescales that we find in our simulations for large KBOs is in agreement with results from previous coagulation simulations (Kenyon \& Luu 1999; Kenyon 2002; Kenyon \& Bromley 2004).

\subsubsection{Different Values of $\alpha$}

Since our analytic result for the mass ratio between large and small KBOs (i.e., $\Sigma / \sigma \sim \alpha^{3 / 4}$ ) can be expressed as a function of $\alpha$ alone, we performed an additional set of coagulation simulations for a value of $\alpha$ that was decreased by a factor of 100. Figure 6 shows the coagulation results for $\alpha=10^{-4}$ (dashed lines), which is roughly the value for the Kuiper Belt, and $\alpha=10^{-6}$ (solid lines). We increased the initial planetesimal mass in the $\alpha=10^{-6}$ simulation by a factor of $10^{1.5}$ to speed up the planetesimal growth. Due to this mass increase, the resulting mass per logarithmic mass interval in large bodies should be the same in both simulations. This is indeed what we see in Figure 6, confirming our analytic expression for $\Sigma$. Figure 6 also shows that the equilibrium growth state, where growth by accretion of small bodies is comparable to the growth due to similar sized mergers, is reached later for $\alpha=10^{-6}$ compared to $\alpha=10^{-4}$. We confirmed that this is not caused by the increased planetesimal mass that was used in the $\alpha=10^{-6}$ simulation. 


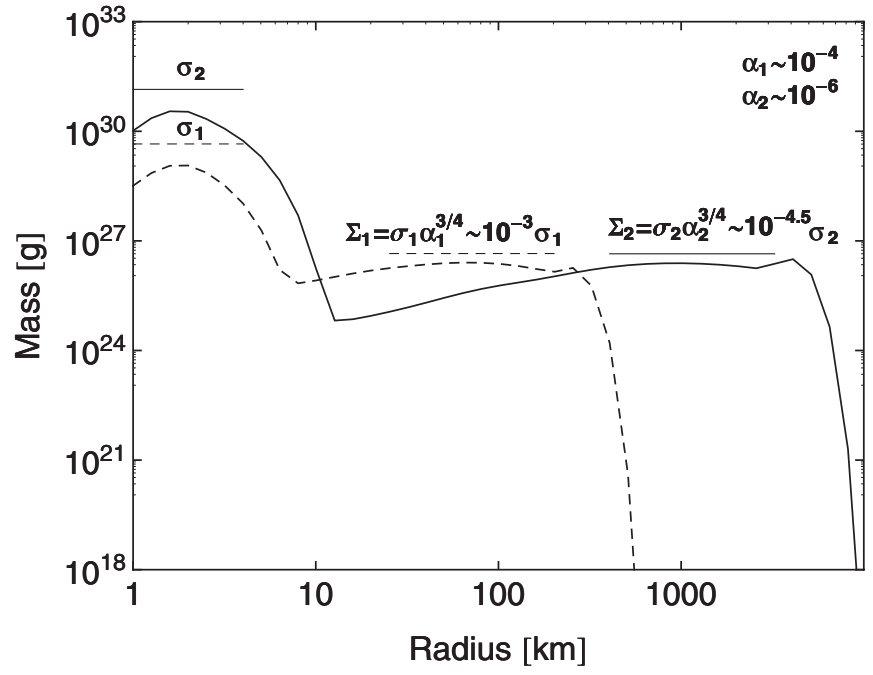

Figure 6. Comparison of our coagulation results for two different values of $\alpha$. The dashed lines correspond to $\alpha_{1}=10^{-4}$, which is roughly the value for the Kuiper Belt, and the solid lines correspond to $\alpha_{2}=10^{-6}$, which could, for example, represent a debris disk around a solar mass star with a semimajor axis about 100 times larger than the Kuiper Belt. We increased the initial planetesimal mass in the $\alpha_{2}=10^{-6}$ simulation by a factor of $10^{1.5}$ to speed up the planetesimal growth. The $y$-axis corresponds to the mass in a given $\log _{2}$ mass interval; $\sigma_{1}$ and $\sigma_{2}$ are the sum of the total mass in small planetesimals. $\Sigma_{1}$ and $\Sigma_{2}$ correspond to the mass in large KBOs in a given $\log _{2}$ mass interval and their predicted values from Section 2 are given by the lines drawn below their respective symbols. The agreement between theory and simulation for both systems is clearly shown in Figure 6.

Since $\alpha \sim a^{-1}$, different values of $\alpha$ can be interpreted as corresponding to different semi-major axes. Therefore, the formation of protoplanets located at $20 \mathrm{AU}$, for example, follows a similar runaway growth compared to protoplanets located $40 \mathrm{AU}$, i.e., their size distribution develops the same power law, with roughly equal mass per logarithmic mass bin, as we derived for the current location of the Kuiper Belt, but with a different value of $\Sigma / \sigma$. Since $\Sigma / \sigma \sim \alpha^{3 / 4}$, we have that at 20 $\mathrm{AU} \Sigma$ is about a factor of $2^{3 / 4}$ larger compared to $\Sigma$ at $40 \mathrm{AU}$. This implies that at $20 \mathrm{AU}$ more of the total initial planetesimal mass is converted into large objects during runaway growth compared to at $40 \mathrm{AU}$, but this overall increase in $\Sigma$ is less than a factor of two. The absolute value of $\Sigma$ is also likely larger at $20 \mathrm{AU}$ compared to at $40 \mathrm{AU}$. For an MMSN-type disk, which has a radial surface density profile that scales as $a^{-3 / 2}$, we have that $\Sigma$ at $20 \mathrm{AU}$ is about $2^{9 / 4} \sim 5$ times larger than the corresponding value at $40 \mathrm{AU}$. The formation timescale, $\tau$, of Pluto-sized objects can be written as

$$
\tau^{-1} \sim \Omega \frac{\sigma}{\rho R} \alpha^{-3 / 4}
$$

where we started from Equation (8) and used $\Sigma / \sigma \sim \alpha^{3 / 4}$. Therefore, for $R \sim R_{\text {Pluto }}$ and an MMSN-type disk with an $a^{-3 / 2}$ radial surface density profile, we find that $\tau_{\text {Pluto }} \propto a^{9 / 4}$. The formation timescale for Pluto-sized objects is therefore about $2^{9 / 4} \sim 5$ times shorter at $20 \mathrm{AU}$ compared to $40 \mathrm{AU}$.

\section{COLLISIONS AND OTHER CONSIDERATIONS}

We have so far neglected collisions among the small bodies. In this section, we first derive the planetesimal-planetesimal collision timescale and show that planetesimal collisions can be neglected during the KBO growth for planetesimal sizes of about a kilometer and larger. We then discuss how our results would be altered, if planetesimal collisions had been important during the KBO growth. In addition, we examine the effect of semi-collisional accretion (Sari \& Goldreich 2006; Schlichting $\&$ Sari 2007) and frequent binary mergers on the growth of KBOs.

The planetesimal-planetesimal collision time for objects with radius, $r$, and with a velocity dispersion that has been excited above their escape velocity is given by

$$
\tau_{\text {Coll }} \sim \Omega^{-1} \frac{\rho r}{\sigma} .
$$

Comparing Equation (31) to the Pluto formation timescale, which is given by the inverse of Equation (8) we find that

$$
\frac{\tau_{\text {Coll }}}{\tau_{\text {Pluto }}} \sim\left(\frac{\Sigma r}{\sigma R_{\text {Pluto }}}\right) \alpha^{-3 / 2}
$$

which is $\sim 1$ for 1-km-sized planetesimals and $\Sigma \sim \alpha^{3 / 4} \sigma .1 \mathrm{~km}$ is about the size that planetesimals are expected to have, if they formed by gravitational instability without dissipation of internal angular momentum (Goldreich \& Ward 1973; Goldreich et al. 2004b). This implies that if KBOs grew from a population of 1-km-sized planetesimals then mutual planetesimal collisions become important only once KBOs comparable to the size of Pluto have formed.

If planetesimal-planetesimal collisions are important, then they would, most likely, break up the planetesimals and lead to a damping of the small bodies velocity dispersion, which would therefore no longer grow as $R$, as we assumed in Section 2 . Instead, it would be set by balancing the dynamical stirring from large bodies by collisional cooling. This yields

$$
\frac{u}{v_{H}} \sim \frac{\Sigma s}{\sigma R} \alpha^{-2}
$$

for $u<v_{H}$, where $s$ is the radius of the small bodies (Goldreich et al. 2004b). We note here that $u<v_{H}$ is most likely the relevant case to consider, since $u$ is only slightly super-Hill when collisional cooling is neglected (see Section 2). In this case, the growth of KBOs is given by

$$
\frac{1}{R} \frac{d R}{d t} \sim \Omega \frac{\sigma}{\rho R} \alpha^{-1}\left(\frac{u}{v_{H}}\right)^{-1}+\Omega \frac{\Sigma}{\rho R} \alpha^{-3 / 2},
$$

where we assumed that $\alpha^{1 / 2} v_{H}<u<v_{H}$. Substituting for $u$ from Equation (33) and comparing the two growth rates in Equation (34), we find that the two growth rates become equal when

$$
\frac{\Sigma}{\sigma} \sim\left(\frac{R}{s}\right)^{1 / 2} \alpha^{5 / 4} \sim 10^{-1}\left(\frac{R / 1000 \mathrm{~km}}{s / 1 \mathrm{~cm}}\right)^{1 / 2} .
$$

This implies, that in this case, the growth of large bodies is dominated by the accretion of small bodies until more mass is converted into $\Sigma$, compared to the case without frequent planetesimal collisions. The total mass in large KBOs and the slope of their size distribution are therefore likely to be different from our results in Section 2 and 3, if collisions were important. The KBO growth would be halted, if collisions lead to the onset of a collisional cascade and if the small collisional fragments are efficiently lost from the Kuiper Belt.

So far, we have treated the planetesimal accretion as collisionless, meaning that collisions among planetesimals can be 
neglected while they are inside the Hill sphere of a growing KBO. This assumption is valid, if KBOs formed from $\mathrm{km}$-sized planetesimals. However, if planetesimals are of the order of a meter in size or smaller, either because they formed small, or because they were broken into small pieces, then they are more likely to collide with each other inside a KBO's Hill sphere than to accrete onto the growing KBO directly (Schlichting \& Sari 2007). We call this semi-collisional accretion. Such planetesimal collisions inside the Hill sphere lead to the formation of an accretion disk around the KBOs. If KBOs grew by semicollisional accretion than their growth could have been very fast, because the effective radius for accretion, in this case, is of the order of their Hill sphere. The KBO growth would be dominated by the accretion of small planetesimals for much longer in the semi-collisional regime, compared to the growth scenario discussed and investigated in Sections 2 and 3.

Finally, the growth of KBOs might also have been aided by merging comparable mass Kuiper Belt binaries. As we discuss in Section 5 in detail, a significant fraction of KBOs reside in comparable mass binary systems and such systems likely formed by dynamical processes such as by three-body capture, or by binary formation aided by dynamical friction (Weidenschilling 2002; Goldreich et al. 2002; Funato et al. 2004; Astakhov et al. 2005; Lee et al. 2007). Once formed, the mutual semi-major axis of such binary systems starts to shrink due to dynamical friction generated by the small planetesimals (Goldreich et al. 2002). This eventually leads to merging between the binary components. This channel of growth could be important, because the binary formation time and the time it takes for a binary to spiral in until contact occurs are comparable to the timescale on which large KBOs grow (Goldreich et al. 2002). If merging of binary components is important in the overall KBO growth, then the growth rate would be enhanced compared to Equation (7). This results in a reduced mass surface density for large KBOs, but the power-law slope of the large $\mathrm{KBO}$ size distribution will remain unchanged.

\section{IMPLICATIONS FOR KUIPER BELT BINARIES}

Our coagulation results also have interesting implications for the formation of Kuiper Belt binaries. A significant fraction of KBOs are part of a binary system. The binary fraction varies for different dynamical classes and it is highest in the cold classical belt (Noll et al. 2008c), where it is about 30\%. More than 70 binaries have been discovered in the Kuiper Belt to date and their number continues to rise. High-mass ratio binary systems, including Pluto/Charon, likely formed in a collision and subsequent tidal evolution. However, the majority of Kuiper Belt binaries consist of comparable mass companions with wide separations and have too much angular momenta to have formed by the same mechanism. Instead these systems most likely have a dynamical origin like, for example, binary formation by threebody capture, or binary formation aided by dynamical friction (Weidenschilling 2002; Goldreich et al. 2002; Funato et al. 2004; Astakhov et al. 2005; Lee et al. 2007). The Hill sphere, the region interior to an object's Hill radius, sets the maximum phase space available for binary formation by such dynamical processes. Dynamical binary formation scenarios take advantage of the increased size of the Hill radius, which is more than an order of magnitude larger in the Kuiper Belt than for similar sized objects in the Asteroid belt and therefore make the Kuiper Belt the ideal place for the formation of wide, comparable mass binaries.

Dynamical binary formation scenarios require $v^{\prime}<v_{H}$ for efficient formation, since binary formation rates quickly

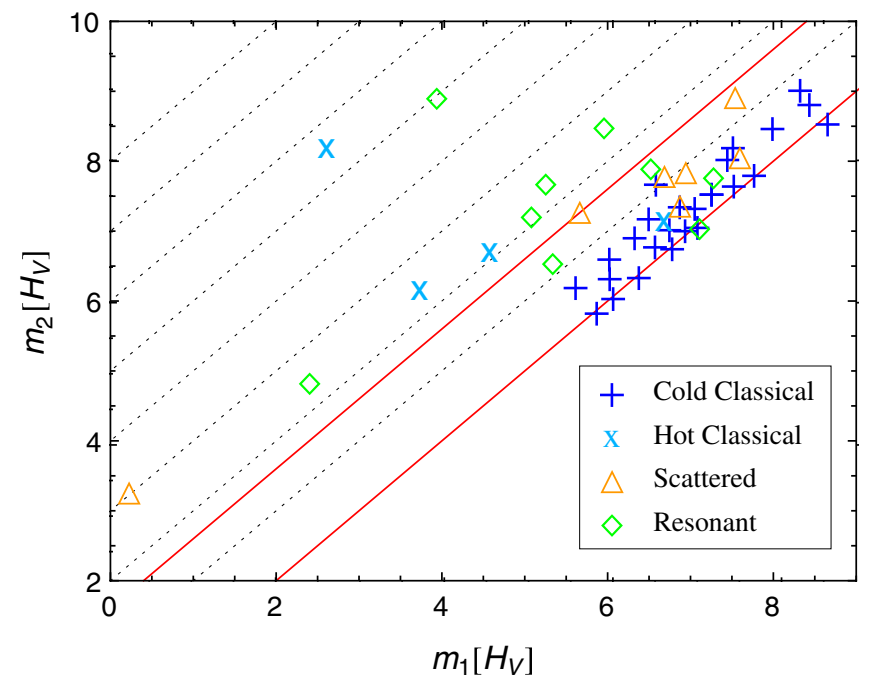

Figure 7. $H_{V}$ magnitudes of both components of Kuiper Belt binaries, labeled by their respective dynamical class according to the classification by Gladman et al. (2008). The black dotted lines, correspond to increments of 1 mag difference between the binary components. The region enclosed by the solid red lines ranges from equal magnitude binary components to a maximum magnitude difference of $\Delta_{\text {mag }} \sim 1$.7. This corresponds to the maximum size difference that Kuiper Belt binary components with similar albedos can have as predicted by this work, if binary formation preceded by dynamical processes (see Section 5 for details). All binaries in the cold classical belt lie in the region enclosed by the solid red lines. In contrast to the cold classical population, the binary components of other dynamical classes span a wider range in mass ratios. The plotted binary data were obtained from the following references: Noll et al. (2008a) and references therein, Noll et al. (2008c), and Lin et al. (2010). We calculated the $H_{V}$ magnitude of each binary component from the magnitude difference between the primary and secondary and from their combined $H_{V}$ magnitude as given in the references above. Pluto and Eris, which are both not part of the classical belt, are not shown on this plot, since they have $H_{V}$ magnitudes brighter than 0 and fall therefore to the left of this figure.

(A color version of this figure is available in the online journal.)

exceed the age of the solar system, once the velocity dispersion significantly exceeds the Hill velocity (Noll et al. 2008a; Schlichting \& Sari 2008). Since KBO growth and binary formation occur concurrently, we can predict the maximum mass ratio for such binary systems because we know the range of $\mathrm{KBO}$ radii that have sub-Hill velocities at any given stage during the growth. From Equation (15) we have $v^{\prime}<v_{H}$ for KBOs with radii greater than $R^{\prime} \sim \alpha^{1 / 12} R \sim 0.5 R$. This implies a maximum mass ratio of $\sim \alpha^{-1 / 4} \sim 10$ between the primary and secondary and translates into a maximum magnitude difference between the binary components, assuming similar albedos, of about $\Delta_{\text {mag }} \sim 1.7$. This provides an explanation for the strong clustering in $\Delta_{\mathrm{mag}}$ of binaries in the cold classical belt. This clustering was first pointed out by Noll et al. (2008b) and is shown in Figure 7. The figure shows that all binaries in the cold classical belt (defined here as classical KBOs with inclinations less than 5.5) have $\Delta_{\text {mag }}<1.7$, i.e., they lie in the region enclosed by the solid red lines. Our coagulation work therefore suggests that the observed binaries in the classical belt formed by dynamical processes during the growth of the KBOs themselves and that this formation most likely took place in situ. The cold classical belt is therefore not only distinct in its inclination distribution (Brown 2001; Elliot et al. 2005; Gulbis et al. 2010), color (Tegler \& Romanishin 2000; Trujillo \& Brown 2002; Petit et al. 2009) and binary fraction (Stephens \& Noll 2006), but also in terms of the type of its binaries, i.e., they are exclusively comparable mass ratio binaries with $\Delta_{\text {mag }}<1.7$ (Noll et al. 2008a, 2008b). Figure 7 shows that, in contrast to the 
cold classical population, other dynamical classes have binaries with a wide range of mass ratios and they therefore most likely contain dynamically and collisionally formed binary systems. The fact that the other dynamical classes have comparable mass binaries as well as systems with a wider range of component sizes is consistent with the idea that they are a superposition of two original populations, namely a dynamical cold (low inclination) population that formed close to its current location and a dynamical hot (high inclination) population that formed closer to the Sun. Kuiper Belt binaries may therefore prove to be useful probes for entangling the two original dynamical populations, if they existed (Murray-Clay \& Schlichting 2010).

\section{DISCUSSION \& CONCLUSIONS}

We carried out an analytic and numerical investigation of runaway growth of protoplanets with a focus on the Kuiper Belt. Since the Kuiper Belt is a remnant of the primordial solar system where planet formation never reached completion, it contains some of the least processed bodies. As a result, it provides a snapshot of earlier stages of planet formation, which were erased elsewhere in the solar system where planet formation proceeded to completion. Our results for runaway growth, which we summarize below, therefore, do not only apply to the Kuiper Belt, but also to planet formation before the onset of oligarchic growth. In addition, our findings apply to protoplanetary growth in debris disks around other stars, as long as the protoplanets' growth time is shorter than the planetesimal-planetesimal collision time, such that dynamical cooling and possible mass loss due to frequent planetesimal collisions can be neglected.

In this paper, we presented analytic work that describes the growth of KBOs, the evolution of their velocity dispersion, and that provides insights into the underlying physical processes that give rise to the KBO size distribution. Our work successfully explains the observed slope of the KBO size distribution as well as the total mass that is present in large KBOs today. In addition, it predicts the maximum mass ratio of Kuiper Belt binaries that formed by dynamical processes, which explains the observed clustering in binary companion sizes that are seen in the cold classic belt. We confirmed our analytic results with numerical coagulation simulations.

We find that the KBO growth proceeds as follows. Initially, all the mass resides in small planetesimals and large KBOs start to form by accreting small planetesimals. This growth continues until growth by merging with comparable sized KBOs become comparable to growth by accreting small bodies. We show that this condition sets in when $\Sigma / \sigma \sim \alpha^{3 / 4} \sim 10^{-3}$. From that time onward, the growth and the evolution of the velocity dispersion become self-similar and $\Sigma$ remains roughly constant, since the increase in $\Sigma$ by the accretion of small planetesimals is balanced by a decease due to the accretion of large bodies. We showed that this mode of growth leads to a KBO size distribution with a power-law index $q=4$. This is in good agreement with observations of the Kuiper Belt size distribution, which is well described by a power law with an index that is consistent with $q=4$ within $1 \sigma$ (e.g., Trujillo et al. 2001; Bernstein et al. 2004; Fuentes \& Holman 2008; Fraser et al. 2008). A single albedo is assumed for all sizes when converting from the observed magnitude distribution to the KBO size distribution. Therefore, possible albedo variations as a function of size could introduce a significant uncertainty in the estimate of $q$. The best-fit value for the power-law index is typically found to somewhat exceed $q=4$. We note that this could be due to fitting the high end of the size distribution that corresponds to the largest KBOs, which is somewhat steeper than the rest of the distribution (see Figure 1). This steeper end of the KBO size distribution is due to the fact that the largest objects did not have enough time to grow to their steady-state abundance.

If KBOs formed by coagulation from kilometer-sized planetesimals, then there could not have been significantly more mass in large KBOs than what is observed today, unless the MMSN was initially enhanced by several orders of magnitude. This result is in good agreement with the current mass in large KBOs, since it is about $\Sigma / \sigma \sim \alpha^{3 / 4} \sim 10^{-3}$ of the MMSN that was enhanced by a factor of a few, as required for the formation of Uranus and Neptune. The observed mass in large KBOs and their size distribution therefore support the hypothesis that KBOs formed from an MMSN-type disk by coagulation from kilometer-sized planetesimals. The growth of KBOs ended in this runaway phase with $\Sigma \sim \alpha^{3 / 4} \sigma \sim 10^{-3} \sigma$. In the context of planet formation, this phase of runaway growth was terminated by the onset of oligarchic growth when each large body dominates the stirring in its own feeding zone. During oligarchic growth $\Sigma$ continues to grow until it becomes comparable to $\sigma$.

Our understanding of the growth, the value of $\Sigma / \sigma$, and the size distribution of large bodies differs from previous works that investigated the details of runaway growth (Makino et al. 1998; Lee 2000; Malyshkin \& Goodman 2001). This is mainly due to the fact that these works did not take into account the simultaneous evolution of the velocity dispersion during the growth, but assumed either equipartition between the different mass bins (Makino et al. 1998; Malyshkin \& Goodman 2001) and/or a constant velocity dispersion for all sizes (Lee 2000).

Since efficient binary formation by dynamical processes can only proceed among KBOs that have sub-Hill velocity dispersions (Noll et al. 2008a; Schlichting \& Sari 2008) and because we follow the evolution of the KBO velocity dispersion as well as their growth, we can predict the maximum mass ratio for such binary systems. Our work yields a maximum binary mass ratio of $\alpha^{-1 / 4} \sim 10$, or, in other words, a maximum magnitude difference between binary components, assuming similar albedos, of $\Delta_{\mathrm{mag}} \sim 1.7$. This explains the clustering in $\Delta_{\text {mag }}$ of all the observed Kuiper Belt binaries that are part of the cold classic belt, which make up about half of all known binary systems in the Kuiper Belt.

There exists some tentative observational evidence that the power-law size distribution might be different for KBOs with inclinations greater and smaller than $\sim 5^{\circ}$ (Bernstein et al. 2004; Fuentes \& Holman 2008; Fraser et al. 2010). However, Fraser \& Kavelaars (2009) and Fuentes et al. (2010) found no conclusive evidence supporting such a difference. It will be interesting to see the results of future KBO surveys that address this question. Should there indeed exist a difference between various dynamical classes then this could have very interesting implications for the formation and collisional evolution of the Kuiper Belt.

We thank Peter Goldreich and Margaret Pan for helpful comments on this manuscript. For H.S., support for this work was provided by a Canadian Institute for Theoretical Astrophysics Postdoctoral Fellowship and by NASA through Hubble Fellowship grant No. HST-HF-51281.01-A awarded by the Space Telescope Science Institute, which is operated by the Association of Universities for Research in Astronomy, Inc., for NASA, under contact NAS 5-26555. The research of R.S. is supported by ERC, IRG, and $H S T$ grants. 


\section{REFERENCES}

Astakhov, S. A., Lee, E. A., \& Farrelly, D. 2005, MNRAS, 360, 401

Bernstein, G. M., Trilling, D. E., Allen, R. L., Brown, M. E., Holman, M., \& Malhotra, R. 2004, AJ, 128, 1364

Bianco, F. B., et al. 2010, AJ, 139, 1499

Brown, M. E. 2001, AJ, 121, 2804

Chiang, E., Lithwick, Y., Murray-Clay, R., Buie, M., Grundy, W., \& Holman, M. 2007, in Protostars and Planets V, ed. B. Reipurth, D. Jewitt, \& K. Keil (Tucson, AZ: Univ. Arizona Press), 895

Davis, D. R., \& Farinella, P. 1997, Icarus, 125, 50

Dodson-Robinson, S. E., \& Bodenheimer, P. 2010, Icarus, 207, 491

Dohnanyi, J. W. 1969, J. Geophys. Res., 74, 2531

Dones, L., \& Tremaine, S. 1993, Icarus, 103, 67

Elliot, J. L., et al. 2005, AJ, 129, 1117

Fraser, W. C., Brown, M. E., \& Schwamb, M. E. 2010, Icarus, 210, 944

Fraser, W. C., \& Kavelaars, J. J. 2009, AJ, 137, 72

Fraser, W. C., et al. 2008, Icarus, 195, 827

Fuentes, C. I., George, M. R., \& Holman, M. J. 2009, ApJ, 696, 91

Fuentes, C. I., \& Holman, M. J. 2008, AJ, 136, 83

Fuentes, C. I., Holman, M. J., Trilling, D. E., \& Protopapas, P. 2010, ApJ, 722, 1290

Funato, Y., Makino, J., Hut, P., Kokubo, E., \& Kinoshita, D. 2004, Nature, 427, 518

Gladman, B., Marsden, B. G., \& Vanlaerhoven, C. 2008, in Nomenclature in the Outer Solar System, ed. M. A. Barucci et al. (Tucson, AZ: Univ. of Arizona Press), 43

Goldreich, P., Lithwick, Y., \& Sari, R. 2002, Nature, 420, 643

Goldreich, P., Lithwick, Y., \& Sari, R. 2004a, ApJ, 614, 497

Goldreich, P., Lithwick, Y., \& Sari, R. 2004b, ARA\&A, 42, 549

Goldreich, P., \& Ward, W. R. 1973, ApJ, 183, 1051

Greenberg, R., Bottke, W. F., Carusi, A., \& Valsecchi, G. B. 1991, Icarus, 94, 98

Greenberg, R., Hartmann, W. K., Chapman, C. R., \& Wacker, J. F. 1978, Icarus, 35,1

Gulbis, A. A. S., Elliot, J. L., Adams, E. R., Benecchi, S. D., Buie, M. W., Trilling, D. E., \& Wasserman, L. H. 2010, AJ, 140, 350

Hayashi, C. 1981, Prog. Theor. Phys. Suppl., 70, 35

Jewitt, D., \& Luu, J. 1993, Nature, 362, 730

Kenyon, S. J. 2002, PASP, 114, 265

Kenyon, S. J., \& Bromley, B. C. 2004, AJ, 128, 1916
Kenyon, S. J., \& Luu, J. X. 1999, AJ, 118, 1101

Kokubo, E., \& Ida, S. 1996, Icarus, 123, 180

Lee, E. A., Astakhov, S. A., \& Farrelly, D. 2007, MNRAS, 379, 229

Lee, M. H. 2000, Icarus, 143, 74

Lin, H., Kavelaars, J. J., Ip, W., Gladman, B. J., Petit, J. M., Jones, R. L., \& Parker, J. W. 2010, PASP, 122, 1030

Liu, C.-Y., Chang, H.-K., Liang, J.-S., \& King, S.-K. 2008, MNRAS, 388, L44

Makino, J., Fukushige, T., Funato, Y., \& Kokubo, E. 1998, New Astron., 3, 411

Malyshkin, L., \& Goodman, J. 2001, Icarus, 150, 314

Murray-Clay, R., \& Schlichting, H. E. 2010, submitted

Noll, K. S., Grundy, W. M., Chiang, E. I., Margot, J.-L., \& Kern, S. D. 2008a, in Binaries in the Kuiper Belt, ed. M. A. Barucci et al. (The Solar System Beyond Neptune; Tucson, AZ: Univ. Arizona Press), 345

Noll, K. S., Grundy, W. M., Kern, S. D., \& Barker, E. A. 2008b, LPI Contrib. 1405,8298

Noll, K. S., Grundy, W. M., Stephens, D. C., Levison, H. F., \& Kern, S. D. 2008c, Icarus, 194, 758

Pan, M., \& Sari, R. 2005, Icarus, 173, 342

Petit, J.-M., Kavelaars, J., Gladman, B., \& Laredo, T. 2008, in Size Distribution of Multikilometer Transneptunian Objects, ed. M. A. Barucci et al. (The Solar System Beyond Neptune; Tucson, AZ: Univ. Arizona Press), 71

Petit, J.-M., et al. 2009, BAAS, 41, 562

Rafikov, R. R. 2003, AJ, 125, 942

Safronov, V. S. 1969, Evolution of the Protoplanetary Cloud and Formation of the Earth and Planets (Translation 1972, NASA TT F-677; Moscow: Nauka)

Sari, R., \& Goldreich, P. 2006, ApJ, 642, L65

Schlichting, H. E., Ofek, E. O., Wenz, M., Sari, R., Gal-Yam, A., Livio, M., Nelan, E., \& Zucker, S. 2009, Nature, 462, 895

Schlichting, H. E., \& Sari, R. 2007, ApJ, 658, 593

Schlichting, H. E., \& Sari, R. 2008, ApJ, 673, 1218

Stephens, D. C., \& Noll, K. S. 2006, AJ, 131, 1142

Stern, S. A. 1996, AJ, 112, 1203

Stern, S. A., \& Colwell, J. E. 1997, ApJ, 490, 879

Tegler, S. C., \& Romanishin, W. 2000, Nature, 407, 979

Trujillo, C. A., \& Brown, M. E. 2002, ApJ, 566, L125

Trujillo, C. A., \& Brown, M. E. 2003, Earth Moon Planets, 92, 99

Trujillo, C. A., Jewitt, D. C., \& Luu, J. X. 2001, AJ, 122, 457

Tsiganis, K., Gomes, R., Morbidelli, A., \& Levison, H. F. 2005, Nature, 435, 459

Weidenschilling, S. J. 2002, Icarus, 160, 212

Wetherill, G. W., \& Stewart, G. R. 1989, Icarus, 77, 330 